\title{
The Low Frequency Approximation of the Sound Radiation Power of Two Vibrating Circular Pistons Embedded in Two Different Rigid Planes of a Three-wall Corner
}

\author{
W.P. RdZAneK*, W.J. RDZAnEK And K. SzEmela \\ Department of Mechatronics and Control Science, Faculty of Mathematics and Natural Sciences \\ University of Rzeszów, Prof. St. Pigonia 1, 35-310 Rzeszów, Poland
}

\begin{abstract}
The problem of sound radiation by a system consisting of two vibrating circular pistons embedded in two of three different planes perpendicular to one another forming a three-wall corner is considered. The earlier published results dealing with the sound radiation by sources vibrating in a three-wall corner are the basis of analysis. According to the earlier studies, the exact formulae for acoustic power of radiation of two circular pistons are used. The formulae are expressed as double Fourier integrals. The active and reactive, self and mutual, components are separated from them as well as the corresponding expressions of the acoustic power of mirror images of the piston sources. The acoustic power of the two sources are expressed in the form of the Rayleigh formulae whereas, in the case of the mirror images, it is expressed in the form of the single series expansion containing spherical Bessel and Neumann functions. In the case of the mutual acoustic power of the sources, approximate formulae are presented for low frequencies. On the basis of the results obtained, the corresponding formulae valid for a two-wall corner are presented as the limiting transitions. All the results presented can be useful, e.g. in designing the room acoustics and outdoor system everywhere the free field conditions are disturbed by the acoustic waves reflected at rigid vertical walls for the wavelengths being considerably shorter than the geometric sizes of the walls.
\end{abstract}

DOI: 10.12693/APhysPolA.125.A-135

PACS: 43.20.Bi, 43.20.El, 43.20.Fn, 43.20.Rz

\section{Introduction}

The problems of sound radiation by vibrating surface sources embedded in rigid baffles as well as the problems of acoustic waves scattering on the obstacles in the vicinity of such rigid baffles are analyzed by scientists throughout the world. A number of studies were published so far, dealing with such phenomena appearing in the regions partially limited by a single baffle, most often being flat, cylindrical, or spherical. Hasheminejad and Alibakhshi have undertaken the problem of the ultrasound scattering on compressible cylinders [1]. Leppington has solved the problem of sound radiation by a convex cylinder in the interval of short waves [2]. Lee and Eom considered the incident acoustic wave transmission through an aperture in a rigid wall penetrated by electric cable of circular cylindrical cross-section. For this purpose, they solved the system of three coupled wave equations [3]. Levine and Leppington developed the effective acoustic damping factor of vibrations of the clamped circular plate [4]. They included the backward effect of the fluid on the plate's vibrations. Hashimoto, Arenas and Crocker applied the resistance matrix method to obtain the acoustic radiation efficiency of vibrating flat circular, annular, and rectangular plates based on their vibration velocity surface distribution [5-7]. Gorazd et al. examined the acoustic pressure field inside a cylindrical duct [8]. Measurements of the acoustic pressure were carried out for two types

*corresponding author; e-mail: wprdzank@ur.edu.pl of hard-walled cylindrical ducts corresponding to models known as the infinite duct and the semi-infinite duct. The axisymmetric modes were excited only and good conformity between measurements and theoretical predictions was achieved in the frequency interval of $0.8-5.5 \mathrm{kHz}$. Snakowska has presented the spatial distribution of energy radiated from the outlet of a cylindrical duct in case of multimodal excitation [9]. The semi-infinite unflanged hard duct was considered together with the Neumann boundary conditions. The solutions were obtained using the Wiener-Hopf method. The theoretical results were proposed and the numerical analysis presented for the acoustic power radiated outside the duct. Mellow has proposed an interesting method to obtain a low frequency approximation of a vibrating resilient disk embedded in a rigid baffle [10]. First, he transformed the integration path in the plane of complex variable for the acoustic power expression, and then used the King integral and the Gegenbauer summation theorem together with the Lommel expansion to obtain a relatively compact analytic solution for the axisymmetric radiation useful for numerical calculations. The computations of the sound power radiated by a plane vibrating structure were based by Arenas [11] on the surface velocity information only. For this purpose he used the method of the radiation resistance matrix of the structure. He applied his method to the sound power of the structural axisymmetric modes of clamped and simply-supported circular baffled plates.

A number of sound and vibration canceling algorithms were developed achieving a significant decrease in both vibrations and sound power radiated. Leniowska located 
symmetrically a system of piezoelectric actuators and applied a linear state model to develop the control algorithm including the effects of viscous damping of the plate as well as the fluid damping [12]. She has obtained a significant reduction of the amplitude of the plate's vibration velocity. Wiciak performed a similar experiments using five piezoelectric elements attached to a clamped aluminum plate [13]. One of the elements was applied as the excitation, two of them as control actuators, and the remaining two as sensors. The structural noise was measured and a significant local noise reduction was achieved, although with an increase in the plate vibrations amplitude distribution. Pawełczyk presented a comprehensive review of recent noise control techniques [14] addressing several specific aspects. He compared single-channel and multi-channel systems and formulated some general requirements of an efficient control system as well as its limitations providing some fundamental examples. Zawieska presented some selected results of analyses and simulations carried out for the development of a system for active reduction of noise radiated by high power electric transformers [15]. His analysis covers mathematical description of the sound radiation by transformer enclosures modeled as vibrating rectangular plates. Additionally, the transformers were modeled by the appropriate arrays of loudspeakers. Brański [16] considered active vibration control of an asymmetric structure such as a vibrating triangular plate. He proposed an algorithm focusing only on the second mode shape of the plate by selecting the appropriate frequency interval. He obtained considerable vibration velocity reduction as well as a good agreement with measurements performed at the experimental setup.

Considerably fewer studies deal with solutions for the regions partially limited by systems of baffles. Hasheminejad and Azarpeyvand analyzed the sound radiation of the vibrating spherical surface located in the vicinity of a two-wall corner [17]. Rdzanek and Rdzanek presented the Green's function for the problem of sound radiation by a vibrating circular source embedded in the region of two-wall corner and three-wall corner [18]. Rdzanek and Szemela analyzed the acoustic power reduction in the system of two vibrating circular pistons in a threewall corner [19]. They limited their considerations to presenting the integral formulations. Szemela, Rdzanek, Rdzanek, and Pieczonka presented the acoustic radiation power of a vibrating circular membrane or plate embedded in the region of a two-wall corner and a three-wall corner [20-22]

\section{The complex acoustic radiation power}

Two circular pistons vibrate harmonically. They are located on the two different planes in the region of the three-wall corner formed by three flat rigid baffles perpendicular to each other (Fig. 1). The local polar coordinate systems are assumed: one with the origin at $\left(x_{01}, y_{01}\right)$, and the other with the origin at $\left(x_{02}, z_{02}\right)$. The radii of the pistons are $a_{1}$ and $a_{2}$, respectively.

As mentioned in the Introduction, integral formulae for the complex acoustic power were presented earlier in [19].
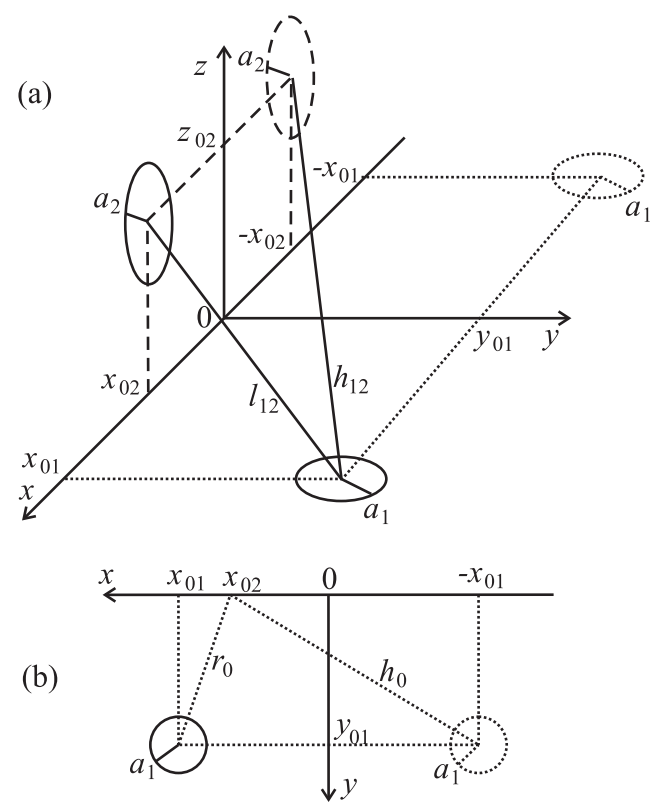

Fig. 1. Arrangement of the two sources and their images: (a) the perspective view; (b) the projection on the $x y$ plane. The solid lines connect the centers of the sources and their images, the dashed lines are drawn on the $x z$ plane, and the dotted lines on the $x y$ plane.

It is obvious, however, that numerical calculations involving integral formulae are considerably time-consuming. Therefore, this study focuses on the low frequency approximation of the self- and mutual acoustic power of radiation. For this purpose, the total acoustic power is expressed as

$$
N=\sum_{i, j=1}^{2} N_{i j}, \quad N_{i j}=\frac{1}{2} \int_{S_{i}} p_{j} v_{i}^{*} \mathrm{~d} S,
$$

where $v^{*} \mathrm{~d} S \equiv \boldsymbol{v}^{*} \cdot \mathrm{d} \boldsymbol{S}=\left(v^{*} \boldsymbol{n}\right) \cdot(\boldsymbol{n} \mathrm{d} S)$ for any surface $S$ enclosing the sources, $S_{1}$ and $S_{2}$ are the areas of pistons of radii $a_{1}$ and $a_{2}$, and $v^{*}$ is the conjugate value of $v$. Since only the surface sources appear that are located at the boundary of the region under consideration, the surface $S$ is selected so that it abuts closely on the sources' surfaces.

If $i=j=1,2$, the acoustic self-power of one of the two considered vibrating pistons is considered. If, on the other hand, $i \neq j$, the acoustic mutual power of the two pistons is regarded. The former is presented in considerable details in [19] and in Appendix. The latter, the time-averaged acoustic mutual power of radiation of the second source under the acoustic pressure radiated by the first source, is formulated as follows (cf. [19])

$$
\begin{aligned}
& N_{21}=8 \varrho_{0} c a_{1} a_{2} v_{1} v_{2}^{*} f_{0}^{\infty} \mathrm{e}^{\mathrm{i} k z_{02} \sqrt{1-\tau^{2}}} J_{1}\left(k a_{1} \tau\right) \\
& \quad \times \frac{F_{21}(\tau) \mathrm{d} \tau}{\sqrt{1-\tau^{2}}},
\end{aligned}
$$

where $p_{1}$ is the acoustic pressure amplitude of the first source exerted at the second source, $v_{1} \equiv v_{01}=\mathrm{const}$, 
$v_{2}=v_{02} \exp (i \delta)$ and $v_{02}=$ const are the vibration velocity amplitudes of the two sources, $f$ is the principal value, and

$$
\begin{aligned}
& F_{21}(\tau)=\int_{0}^{\pi / 2} \bar{f}_{21}(\cos \varphi) \bar{g}(\sin \varphi) \mathrm{d} \varphi \\
& =\int_{0}^{\pi / 2} \bar{f}_{21}(\sin \varphi) \bar{g}(\cos \varphi) \mathrm{d} \varphi, \\
& \bar{f}_{21}(u)=\frac{J_{1}\left(k a_{2} \sqrt{1-\tau^{2} u^{2}}\right)}{\sqrt{1-\tau^{2} u^{2}}} \cos \left(k y_{01} \tau u\right), \\
& \bar{g}(v)=\cos \left(k x_{01} \tau v\right) \cos \left(k x_{02} \tau v\right) .
\end{aligned}
$$

Similarly, the time-averaged acoustic mutual power of radiation of the first source under the acoustic pressure radiated by the second source is formulated as (cf. [19])

$$
\begin{aligned}
& N_{12}=8 \varrho_{0} c a_{1} a_{2} v_{1}^{*} v_{2} f_{0}^{\infty} \mathrm{e}^{\mathrm{i} k y_{01} \sqrt{1-\tau^{2}}} J_{1}\left(k a_{2} \tau\right) \\
& \quad \times \frac{F_{12}(\tau) \mathrm{d} \tau}{\sqrt{1-\tau^{2}}},
\end{aligned}
$$

where $p_{2}$ is the acoustic pressure amplitude of the second source exerted at the first source, and

$$
\begin{gathered}
F_{12}(\tau)=\int_{0}^{\pi / 2} \bar{f}_{12}(\cos \varphi) \bar{g}(\sin \varphi) \mathrm{d} \varphi= \\
\int_{0}^{\pi / 2} \bar{f}_{12}(\sin \varphi) \bar{g}(\cos \varphi) \mathrm{d} \varphi, \\
\bar{f}_{12}(u)=\frac{J_{1}\left(k a_{1} \sqrt{1-\tau^{2} u^{2}}\right)}{\sqrt{1-\tau^{2} u^{2}}} \cos \left(k z_{02} \tau u\right),
\end{gathered}
$$

and $\bar{g}(v)$ is defined by Eq. (2d).

Further, we focus on Eq. (2a) and rearrange it by using identity $2 \cos \alpha \cos \beta=\cos (\alpha-\beta)+\cos (\alpha+\beta)$ and the Lommel expansion

$$
\begin{aligned}
& \frac{J_{1}\left(k a_{2} \sqrt{1-\tau^{2} \cos ^{2} \varphi}\right)}{\sqrt{1-\tau^{2} \cos ^{2} \varphi}}= \\
& \sum_{m=0}^{\infty}\left(\frac{k a_{2}}{2}\right)^{m} \frac{J_{m+1}\left(k a_{2}\right)}{m !} \tau^{2 m}(\cos \varphi)^{2 m} .
\end{aligned}
$$

This expansion is used for the integrand in Eq. (2a), since the integrals over intervals $(0,1)$ and $(1, \infty)$ are convergent. The convergence of the integral over interval $(1, \infty)$ is improved by the exponential function $\exp \left(-k z_{02} \sqrt{\tau^{2}-1}\right)$ (the convergence is assured). Thus we obtain

$$
\begin{aligned}
& n_{21}=\frac{N_{21}}{\varrho_{0} c v_{1} v_{2}^{*}}=4 a_{1} a_{2} f_{0}^{\infty} \frac{\mathrm{e}^{\mathrm{i} k z_{02} \sqrt{1-\tau^{2}}}}{\sqrt{1-\tau^{2}}} J_{1}\left(k a_{1} \tau\right) \mathrm{d} \tau \\
& \quad \times \int_{0}^{\pi / 2}\left[\cos \left(k\left|x_{01}-x_{02}\right| \tau \sin \varphi\right)\right. \\
& \left.\quad+\cos \left[k\left(x_{01}+x_{02}\right) \tau \sin \varphi\right]\right] \cos \left(k y_{01} \tau \cos \varphi\right) \\
& \times \frac{J_{1}\left(k a_{2} \sqrt{1-\tau^{2} \cos ^{2} \varphi}\right)}{\sqrt{1-\tau^{2} \cos ^{2} \varphi}} \mathrm{d} \varphi .
\end{aligned}
$$

The following denotation is assumed:

$$
\begin{aligned}
& n_{21}^{(-)}=n_{21}-n_{21}^{(+)}=4 a_{1} a_{2} f_{0}^{\infty} \frac{\mathrm{e}^{\mathrm{i} k z_{02} \sqrt{1-\tau^{2}}}}{\sqrt{1-\tau^{2}}} J_{1}\left(k a_{1} \tau\right) \mathrm{d} \tau \\
& \quad \times \int_{0}^{\pi / 2} \cos \left(k\left|x_{01}-x_{02}\right| \tau \sin \varphi\right) \cos \left(k y_{01} \tau \cos \varphi\right) \\
& \quad \times \frac{J_{1}\left(k a_{2} \sqrt{1-\tau^{2} \cos ^{2} \varphi}\right)}{\sqrt{1-\tau^{2} \cos ^{2} \varphi}} \mathrm{d} \varphi .
\end{aligned}
$$

The quantity $n^{(+)}$is obtained if $\left|x_{01}-x_{02}\right|$ is replaced by $\left(x_{01}+x_{02}\right)$ in Eq. $(5 \mathrm{~b})$. We use the Lommel expansion (4) in the expression for $n_{21}^{(-)}$obtaining

$$
\begin{aligned}
& n_{21}^{(-)}=4 a_{1} a_{2} \sum_{m=0}^{\infty}\left(\frac{k a_{2}}{2}\right)^{m} \frac{J_{m+1}\left(k a_{2}\right)}{m !} \\
& \quad \times \int_{0}^{\infty} \frac{\mathrm{e}^{\mathrm{i} k z_{02} \sqrt{1-\tau^{2}}}}{\sqrt{1-\tau^{2}}} J_{1}\left(k a_{1} \tau\right) \tau^{2 m} \mathrm{~d} \tau \\
& \quad \times \int_{0}^{\pi / 2} \cos \left(k\left|x_{01}-x_{02}\right| \tau \sin \varphi\right) \cos \left(k y_{01} \tau \cos \varphi\right) \\
& \quad \times(\cos \varphi)^{2 m} \mathrm{~d} \varphi .
\end{aligned}
$$

Now, it is convenient to use the following expansion [23]

$$
\begin{aligned}
& \cos ^{2 m} \varphi=\frac{1}{2^{2 m}} \\
& \times\left[\left(\begin{array}{c}
2 m \\
m
\end{array}\right)+2 \sum_{s=0}^{m-1}\left(\begin{array}{c}
2 m \\
s
\end{array}\right) \cos 2(m-s) \varphi\right],
\end{aligned}
$$

where $\left(\begin{array}{c}2 m \\ s\end{array}\right)=\frac{2 m !}{s !(2 m-s) !}$, and then take $1 / 4$ of [24]:

$$
\int_{0}^{2 \pi} \cos (\alpha r \cos \varphi) \cos (\beta r \sin \varphi) \cos n \varphi \mathrm{d} \varphi=
$$

$$
2 \pi \cos n \varphi_{0} J_{n}(\Gamma r)
$$

where $\varphi_{0}=\arcsin (\alpha / \gamma), \gamma=\left(\alpha^{2}+\beta^{2}\right)^{1 / 2}$, to express Eq. (5c) in the form

$$
\begin{aligned}
& \frac{n_{21}^{(-)}}{2 \pi}=J_{1}\left(k a_{2}\right) f_{0,0}+\sum_{m=1}^{\infty}\left(\frac{k a_{2}}{2}\right)^{m} \frac{J_{m+1}\left(k a_{2}\right)}{\left.2^{2 m} m !\right)}(5 \mathbf{f}) \\
& \times\left\{\left(\begin{array}{c}
2 m \\
m
\end{array}\right) f_{m, m}+2 \sum_{s=0}^{m-1}\left(\begin{array}{c}
2 m \\
s
\end{array}\right) \cos \left[2(m-s) \varphi_{0}\right] f_{m, s}\right\},
\end{aligned}
$$

where

$$
\begin{aligned}
& f_{m, s}=f_{0}^{\infty} \frac{\mathrm{e}^{\mathrm{i} k z_{02} \sqrt{1-\tau^{2}}}}{\sqrt{1-\tau^{2}}} J_{1}\left(k a_{1} \tau\right) J_{2(m-s)}\left(k r_{0} \tau\right) \\
& \quad \times \tau^{2 m} \mathrm{~d} \tau
\end{aligned}
$$

and $r_{0}=\left[\left(x_{01}-x_{02}\right)^{2}+y_{01}^{2}\right]^{1 / 2}$ (cf. Fig. 1).

Further, the formula obtained, i.e. Eq. (5f), is used as the basis for analysis of the active and reactive acoustic mutual power of radiation generated by the vibrating pistons located in a three-wall corner, i.e. of $N_{21}=N_{21}^{\prime}-\mathrm{i} N_{21}^{\prime \prime}$ for steady vibrations, for a single circular frequency $\omega$, for the following time dependencies 
$p(\boldsymbol{r}, t)=p(\boldsymbol{r}) \exp (-i \omega t)$ and $v^{*}(\boldsymbol{r}, t)=v^{*}(\boldsymbol{r}) \exp (-i \omega t)$, where $t$ is time. Obviously, the integral in Eq. (3a) is rearranged in a similar way.

\section{The mutual acoustic power of radiation}

Equation (5f) is useful for calculations of the mutual active acoustic power of radiation if the infinite interval of integration in Eq. $(5 \mathrm{~g})$ is limited to the interval $(0,1)$. Additionally, the real component of the exponential function is to be used only giving

$$
\begin{aligned}
& f_{m, s}^{\prime}=\int_{0}^{1} \frac{\cos \left(k z_{02} \sqrt{1-\tau^{2}}\right)}{\sqrt{1-\tau^{2}}} J_{1}\left(k a_{1} \tau\right) \\
& \times J_{2(m-s)}\left(k r_{0} \tau\right) \tau^{2 m} \mathrm{~d} \tau .
\end{aligned}
$$

Since $k a_{1}<k r_{0}$, we use the substitution $1-\tau^{2}=x^{2}$ in Eq. (6) for $f_{0,0}^{\prime}$, and then we use the Lommel expansion

$$
\begin{aligned}
& \frac{J_{1}\left(k a_{1} \sqrt{1-x^{2}}\right)}{\sqrt{1-x^{2}}}=J_{1}\left(k a_{1}\right) \\
& +\sum_{n=1}^{\infty}\left(\frac{k a_{1}}{2}\right)^{n} \frac{J_{n+1}\left(k a_{1}\right)}{n !} x^{2 n}
\end{aligned}
$$

including only the first term. Further, we use the formula [23], p. 722 , Eq. 6.677 .6

$$
\int_{0}^{1} J_{0}\left(k r_{0} \sqrt{1-x^{2}}\right) \cos \left(k z_{02} x\right) \mathrm{d} x=\frac{\sin \left(k l_{12}\right)}{k l_{12}}
$$

where $l_{12}=\left(r_{0}^{2}+z_{02}^{2}\right)^{1 / 2}$ is the distance between the center points of the two pistons (cf. Fig. 1). Now, Eq. (6) assumes the form

$$
\begin{aligned}
& f_{0,0}^{\prime}=J_{1}\left(k a_{1}\right) \frac{\sin \left(k l_{12}\right)}{k l_{12}}+\sum_{n=1}^{\infty}\left(\frac{k a_{1}}{2}\right)^{n} \frac{J_{n+1}\left(k a_{1}\right)}{n !} \\
& \times \int_{0}^{1} J_{0}\left(k r_{0} \sqrt{1-x^{2}}\right) \cos \left(k z_{02} x\right) x^{2 n} \mathrm{~d} x .
\end{aligned}
$$

If $k a_{1} \ll 1$, we neglect small terms of the order of $\left(k a_{1}\right)^{3}$ and higher, which yields

$$
f_{0,0}^{\prime} \simeq \frac{1}{2} k a_{1} \frac{\sin \left(k l_{12}\right)}{k l_{12}} .
$$

If, additionally, $k a_{2} \ll 1$, we obtain

$$
n_{21}^{\prime(-)} \simeq \frac{\pi}{2} k^{2} a_{1} a_{2} \frac{\sin k l_{12}}{k l_{12}}
$$

from Eqs. (5f) and (7d) by neglecting all the small terms of the order of $\left(k a_{1}\right)^{3}$. We obtain also (cf. Fig. 1)

$$
n_{21}^{\prime(+)} \simeq \frac{\pi}{2} k^{2} a_{1} a_{2} \frac{\sin k h_{12}}{k h_{12}}
$$

where $h_{12}$ is the distance between central points of the source 1 and the mirror image of source 2 .

If $k a_{1}, k a_{2} \ll 1$ and $v_{1} v_{2}^{*}$ is real, the active mutual acoustic power (according to Eqs. (5a) and (5b)) assumes the form

$$
\begin{aligned}
& \operatorname{Re} \frac{N_{21}}{\varrho_{0} c v_{1} v_{2}^{*}}=n_{21}^{\prime}=n_{21}^{\prime(-)}+n_{21}^{\prime(+)} \\
& \simeq \frac{\pi}{2} k^{2} a_{1} a_{2}\left(\frac{\sin k l_{12}}{k l_{12}}+\frac{\sin k h_{12}}{k h_{12}}\right) .
\end{aligned}
$$

The active mutual acoustic power of radiation of the source 1 under the acoustic pressure exerted by the source 2 (the basis integral formula was presented in [19]) is calculated in a similar way, and assumes the form

$$
\begin{aligned}
\operatorname{Re} & \frac{N_{12}}{\varrho_{0} c v_{2} v_{1}^{*}}=n_{12}^{\prime} \\
\simeq & \frac{\pi}{2} k^{2} a_{1} a_{2}\left(\frac{\sin k l_{12}}{k l_{12}}+\frac{\sin k d_{12}}{k d_{12}}\right)
\end{aligned}
$$

where $d_{12}$ is the distance between central points of the source 2 and the mirror image of source 1 (cf. Fig. 1). If it is assumed additionally that the phase difference of the vibration velocity amplitude on both pistons is equal to zero, $\delta=0$, then summing up the results given by Eqs. ( $7 \mathrm{~g})$ and $(7 \mathrm{~h})$ yields $\left(d_{12}=h_{12}\right)$

$$
\operatorname{Re} \frac{N_{21}+N_{12}}{\varrho_{0} c v_{1} v_{2}}=\pi k^{2} a_{1} a_{2}\left(\frac{\sin k l_{12}}{k l_{12}}+\frac{\sin k h_{12}}{k h_{12}}\right)
$$

which represents the sum of the active mutual acoustic power of radiation of two vibrating pistons located on two different walls of a three-wall corner valid for small values of the interference parameters $k a_{1}, k a_{2} \ll 1$.

\section{The reactive mutual acoustic power of radiation}

Eqs. (5a), (5b), and (5f) are used as the basis of analysis of the reactive mutual acoustic power where two kinds of integrals are considered, one over interval $(0,1)$ and the other over interval $(1, \infty)$. Now, we consider the imaginary counterpart of the integral in Eq. $(5 \mathrm{~g})$, represented by integrals

$$
\begin{aligned}
& f_{m, s}^{\text {Fin. }}=\int_{0}^{1} \frac{\sin \left(k z_{02} \sqrt{1-\tau^{2}}\right)}{\sqrt{1-\tau^{2}}} J_{1}\left(k a_{1} \tau\right) \\
& \times J_{2(m-s)}\left(k r_{0} \tau\right) \tau^{2 m} \mathrm{~d} \tau
\end{aligned}
$$

and

$$
\begin{aligned}
& f_{m, s}^{\text {Inf. }}=\int_{1}^{\infty} \frac{\mathrm{e}^{-k z_{02} \sqrt{\tau^{2}-1}}}{\sqrt{\tau^{2}-1}} J_{1}\left(k a_{1} \tau\right) \\
& \times J_{2(m-s)}\left(k r_{0} \tau\right) \tau^{2 m} \mathrm{~d} \tau .
\end{aligned}
$$

We substitute $\tau=\sin \vartheta$ in Eq. (8) for $f_{0,0}^{\text {Fin. }}$, use the expansion series of the Bessel function $J_{0}\left(k r_{0} \sin \vartheta\right)$, whereas in the case when $k a_{1} \ll 1$ we approximate the function $J_{1}\left(k a_{1} \tau\right)$ by $k a_{1} \tau / 2$ thus obtaining

$$
\begin{aligned}
& f_{0,0}^{\text {Fin. }}=\frac{k a_{1}}{2} \sum_{n=0}^{\infty}(-1)^{n} \frac{\left(k r_{0} / 2\right)^{2 n}}{(n !)^{2}} \\
& \quad \times \int_{0}^{\pi / 2} \sin \left(k z_{02} \cos \vartheta\right) \sin ^{2 n+1} \vartheta \mathrm{d} \vartheta .
\end{aligned}
$$

After using the formula [23], p. 942, Eq. 8.551.1:

$$
\begin{aligned}
& \boldsymbol{H}_{\nu}(z)=\frac{2\left(\frac{z}{2}\right)^{\nu}}{\sqrt{\pi} \Gamma\left(\nu+\frac{1}{2}\right)} \\
& \times \int_{0}^{\pi / 2} \sin (z \cos \varphi) \sin ^{2 \nu} \varphi \mathrm{d} \varphi
\end{aligned}
$$

valid for $\operatorname{Re} \nu>-1 / 2$, we have 


$$
\begin{aligned}
& f_{0,0}^{\mathrm{Fin} .}=\sqrt{\pi}\left(k a_{1} / 4\right) \sum_{n=0}^{\infty}(-1)^{n} \frac{\left(k r_{0} / 2\right)^{2 n}}{n !} \\
& \times\left(\frac{2}{k z_{02}}\right)^{n+1 / 2} \boldsymbol{H}_{n+1 / 2}\left(k z_{02}\right)
\end{aligned}
$$

where $\boldsymbol{H}_{\nu}(z)$ is the Struve functions of the order $\nu$. This formula is useful for numerical calculations when $z_{02}>r_{0}$ and $k r_{0}<1$.

We will obtain another representation of $f_{0,0}^{\mathrm{Fin}}$ if we use the expansion series of the function $\sin \left(k z_{02} \cos \vartheta\right)$ in Eq. (10a). Then

$$
\begin{aligned}
& f_{0,0}^{\mathrm{Fin} .}=\sqrt{\pi}\left(k a_{1} / 4\right) \sum_{s=0}^{\infty}(-1)^{s} \frac{\left(k z_{02} / 2\right)^{2 s+1}}{\Gamma(s+3 / 2)} \\
& \times\left(\frac{2}{k r_{0}}\right)^{s+1} J_{s+1}\left(k r_{0}\right)
\end{aligned}
$$

where the following formulae are used

$$
\begin{aligned}
& \int_{0}^{\pi / 2}(\cos \vartheta)^{2(s+1)-1}(\sin \vartheta)^{2(n+1)-1} \mathrm{~d} \vartheta= \\
& \frac{1}{2} \frac{s ! n !}{(s+n+1) !}, \\
& \frac{s !}{(2 s+1) !}=\frac{\sqrt{\pi}}{2^{2 s+1} \Gamma(s+3 / 2)} .
\end{aligned}
$$

In contrast with Eq. (10c), Eq. (10d) is useful for numerical calculations when $z_{02}<r_{0}$ and $k z_{02}<1$.

The multiple expansion series, e.g. the Lommel or Gegenbauer expansions, are more complex in numerical calculations in the case of the function $f_{m, m}^{\text {Fin. }}, f_{m, s}^{\text {Fin. and }}$ are not presented herein.

Besides the reactive interactions expressed by the integral over interval $(0,1)$, it is also necessary to consider the reactive interactions expressed by the integral over interval $(1, \infty)$. Eqs. (5f) and $(5 \mathrm{~g})$ are also the basis of analysis. Referring to Eq. (5f) we obtain

$$
\begin{aligned}
& n_{21}^{(-) \text {Inf. }}=n_{21}^{\text {Inf. }}-n_{21}^{(+) \text {Inf. }}=2 \pi J_{1}\left(k a_{2}\right) f_{0,0}^{\text {Inf. }} \\
& \quad+2 \pi \sum_{m=1}^{\infty}\left(\frac{k a_{2}}{2}\right)^{m} \frac{J_{m+1}\left(k a_{2}\right)}{2^{2 m} m !}\left\{\left(\begin{array}{c}
2 m \\
m
\end{array}\right) f_{m, m}^{\text {Inf. }}\right. \\
& \left.+2 \sum_{s=0}^{m-1}\left(\begin{array}{c}
2 m \\
s
\end{array}\right) \cos \left[2(m-s) \varphi_{0}\right] f_{m, s}^{\text {Inf. }}\right\},
\end{aligned}
$$

where functions $f_{0,0}^{\text {Inf. }}, f_{m, m}^{\text {Inf. }}$ and $f_{m, s}^{\text {Inf. }}$ are defined by Eq. (9). The example of integration is presented for the function $f_{m, s}^{\text {Inf. }}$, where substitution $x=\sqrt{\tau^{2}-1}$ is used giving

$$
\begin{aligned}
& f_{m, s}^{\text {Inf. }}=\int_{0}^{\infty} \mathrm{e}^{-k z_{02} x} J_{2(m-s)}\left(k r_{0} \sqrt{x^{2}+1}\right) \\
& \times \frac{J_{1}\left(k a_{1} \sqrt{x^{2}+1}\right)}{\sqrt{x^{2}+1}}\left(x^{2}+1\right)^{m} \mathrm{~d} x .
\end{aligned}
$$

The Bessel function of the first order is expressed using the Lommel expansion (cf. Eq. (4)) whereas the Bessel function of the order $2(m-s)$ is expressed using the
Gegenbauer expansion [23]

$$
\begin{aligned}
& \frac{J_{\nu}\left(a \sqrt{x^{2}+1}\right)}{\left(\sqrt{x^{2}+1}\right)^{\nu}}=\left(\frac{2}{a x}\right)^{\nu} \sum_{r=0}^{\infty}(-1)^{r} \frac{(2 r+\nu) \Gamma(r+\nu)}{r !} \\
& \times J_{2 r+\nu}(a) J_{2 r+\nu}(a x)
\end{aligned}
$$

where $\nu \neq 0,-1,-2, \ldots$ Assuming that $m=1,2, \ldots$, it is obtained that

$$
\begin{aligned}
& f_{m, s}^{\text {Inf. }}=\sum_{q=0}^{\infty}\left(\frac{k a_{1}}{2}\right)^{q} \frac{J_{q+1}\left(k a_{1}\right)}{q !} \sum_{n=0}^{\infty} \sum_{w=0}^{m-s}(-1)^{n+q} \\
& \times\left(\begin{array}{c}
m-s \\
w
\end{array}\right)\left(\frac{2}{k r_{0}}\right)^{2 m-2 s} \frac{(2 n+2 m-2 s)}{n !} \\
& \times \Gamma(n+2 m-2 s) J_{2 n+2 m-2 s}\left(k r_{0}\right) \int_{0}^{\infty} \mathrm{e}^{-k z_{02} x} \\
& \times J_{2 n+2 m-2 s}\left(k r_{0} x\right) x^{2 q+2 w-2 m+2 s} \mathrm{~d} x,
\end{aligned}
$$

where the Newton binomial theorem is used,

$$
\left(x^{2}+1\right)^{m-s}=\sum_{w=0}^{m-s}\left(\begin{array}{c}
m-s \\
w
\end{array}\right) x^{2 w} .
$$

The following integral formula is further used [23]

$$
\begin{aligned}
& \int_{0}^{\infty} \mathrm{e}^{-\alpha x} J_{\nu}(\beta x) x^{\mu-1} \mathrm{~d} x=\Gamma(\nu+\mu)\left(\alpha^{2}+\beta^{2}\right)^{-\mu / 2} \\
& \quad \times P_{\mu-1}^{-\nu}\left(\alpha / \sqrt{\alpha^{2}+\beta^{2}}\right)
\end{aligned}
$$

if $\alpha>0, \beta>0, \operatorname{Re}(\nu+\mu)>0$. Assuming in Eq. (11d) that $\nu=2 n+2 m-2 s>0$, and $\mu=2 q+2 w+2 s-2 m+1$, $\nu+\mu=2 n+2 q+2 w+1>0$, leads to

$$
\begin{aligned}
& f_{m, s}^{\text {Inf. }}=\left(\frac{2}{k r_{0}}\right)^{2 m-2 s} \sum_{q=0}^{\infty}\left(\frac{k a_{1}}{2}\right)^{q} J_{q+1}\left(k a_{1}\right) \\
& \times \sum_{n=0}^{\infty}(-1)^{n+q}(2 n+2 m-2 s) \frac{\Gamma(n+2 m-2 s)}{n !} \\
& \times \frac{\Gamma(2 n+2 q+2 w+1)}{q !} \sum_{w=0}^{m-s}\left(\begin{array}{c}
m-s \\
w
\end{array}\right) \\
& \quad \times \frac{P_{2(q+w+s-m)}^{-2(n+m-s)}\left(z_{02} / \sqrt{z_{02}^{2}+r_{0}^{2}}\right)}{\left[\left(k z_{02}\right)^{2}+\left(k r_{0}\right)^{2}\right]^{q+w+s-m+1 / 2}} \\
& \times J_{2 n+2 m-2 s}\left(k r_{0}\right),
\end{aligned}
$$

where $P_{n}^{m}(x)$ are the associated Legendre polynomials, and $P_{n}^{m}(x)=0$ for $m>n$. The expression in Eq. (11g) can also be presented in another form if the following formula ([23], p. 700, Eq. 6.621.4) is used

$$
\begin{aligned}
& \int_{0}^{\infty} x^{m+1} \mathrm{e}^{-\alpha x} J_{\nu}(\beta x) \mathrm{d} x=(-1)^{m+1} \beta^{-\nu} \frac{\mathrm{d}^{m+1}}{\mathrm{~d} \alpha^{m+1}} \\
& \times\left[\frac{\left(\sqrt{\alpha^{2}+\beta^{2}}-\alpha\right)^{\nu}}{\sqrt{\alpha^{2}+\beta^{2}}}\right]
\end{aligned}
$$

instead of Eq. (11f), where $\beta>0, \operatorname{Re} \nu>-m-2$ and $2 n>-2 q-2 w-1$. 
While calculating the integral in Eq. (9) for $f_{m, m}^{\mathrm{Inf}}$, the substitution $x=\sqrt{\tau^{2}-1}$ is used, the Bessel function of the zero order is expressed using the Lommel expansion whereas the Bessel function of the first order is replaced with its Gegenbauer expansion, and the Newton binomial theorem is used $\left(x^{2}+1\right)^{m}=\sum_{w=0}^{m}\left(\begin{array}{c}m \\ w\end{array}\right) x^{2 m}$. This leads to

$$
\begin{aligned}
& f_{m, m}^{\text {Inf. }}=\frac{2}{k a_{1}} \sum_{q=0}^{\infty} \frac{\left(k r_{0} / 2\right)^{q} J_{q+1}\left(k r_{0}\right)}{q !} \sum_{n=0}^{\infty}(-1)^{n+q} \\
& \times(2 n+1) J_{2 n+1}\left(k a_{1}\right) \sum_{w=0}^{m}\left(\begin{array}{c}
m \\
w
\end{array}\right) \int_{0}^{\infty} \mathrm{e}^{-k z_{02} x} \\
& \times J_{2 n+1}\left(k a_{1} x\right) x^{2 q+2 w-1} \mathrm{~d} x
\end{aligned}
$$

and after using Eq. (11f), to

$$
\begin{aligned}
& f_{m, m}^{\text {Inf. }}=\frac{2}{k a_{1}} \sum_{q=0}^{\infty} \frac{\left(k r_{0} / 2\right)^{q}}{q !} J_{q+1}\left(k r_{0}\right) \sum_{n=0}^{\infty}(-1)^{n+q} \\
& \times(2 n+1) J_{2 n+1}\left(k a_{1}\right) \sum_{w=0}^{m}\left(\begin{array}{c}
m \\
w
\end{array}\right)(2 n+2 q+2 w) ! \\
& \times \frac{P_{2 q+2 w-1}^{-2 n-1}\left(z_{02} / \sqrt{z_{02}^{2}+a_{1}^{2}}\right)}{\left[\left(k z_{02}\right)^{2}+\left(k a_{1}\right)^{2}\right]^{q+w}} .
\end{aligned}
$$

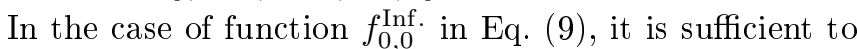
assume that $m=0$ in Eq. (13).

The corresponding contributions to the reactive mutual acoustic power of radiation $\operatorname{Im} N_{21}$ and $\operatorname{Im} N_{12}$ can be obtained using expressions $f_{0}^{\text {Inf. }}, f_{m}^{\text {Inf. }}$, and $f_{m, s}^{\text {Inf. }}$ (cf. $\operatorname{Re} N_{21}$ and $\operatorname{Re} N_{12}$ ).

\section{The calculations of the reactive mutual acoustic power of radiation using the Hilbert transform}

In the case when the interference parameters are small, the sum of the active mutual acoustic powers radiated by the two vibrating pistons (cf. Fig. 1) can be used to calculate the reactive mutual power of the considered sound sources. First, the active mutual acoustic power given by $\mathrm{Eq}$. (7i) is formulated as

$$
\begin{aligned}
& P^{\prime}(k)=\operatorname{Re} \frac{N_{21}+N_{12}}{\frac{\pi}{2} a_{1} a_{2} \varrho_{0} c v_{1} v_{2}} \\
& \quad=2 k^{2}\left(\frac{\sin k l_{12}}{k l_{12}}+\frac{\sin k h_{12}}{k h_{12}}\right)
\end{aligned}
$$

and then the Hilbert transform [25]

$$
P^{\prime \prime}(k)=\frac{1}{\pi} \int_{-\infty}^{+\infty} \frac{P^{\prime}(x) \mathrm{d} x}{x-k}
$$

is used, where $P^{\prime \prime}(x)$ is the imaginary component of the complex function $P=P^{\prime}-$ i $P^{\prime \prime}$.

The normalized active mutual acoustic power of radiation (14) is the even function with respect to the wavenumber $k$. Therefore, the Hilbert transform in Eq. (15) can be expressed as

$$
P^{\prime \prime}(k)=\frac{2 k}{\pi} \int_{0}^{\infty} \frac{P^{\prime}(x) \mathrm{d} x}{x^{2}-k^{2}} .
$$

Now, Eq. (16) is applied to the right hand side of Eq. (14) and the following formula is used [23]

$$
f_{0}^{\infty} \frac{y \sin (b y)}{y^{2}-a^{2}} \mathrm{~d} y=\frac{\pi}{2} \cos (a b)
$$

for $b>0$, giving

$$
\begin{gathered}
P^{\prime \prime}(k)=\operatorname{Im} \frac{N_{21}+N_{12}}{\frac{\pi}{2} a_{1} a_{2} \varrho_{0} c v_{1} v_{2}}= \\
2 k^{2}\left(\frac{\cos k l_{12}}{k l_{12}}+\frac{\cos k h_{12}}{k h_{12}}\right) .
\end{gathered}
$$

Finally, the complex acoustic power of radiation can be approximated by

$$
P=P^{\prime}-\mathrm{i} P^{\prime \prime}=2 k^{2}\left(\frac{\mathrm{e}^{\mathrm{i} k l_{12}}}{\mathrm{i} k l_{12}}+\frac{\mathrm{e}^{\mathrm{i} k h_{12}}}{\mathrm{i} k h_{12}}\right) .
$$

\section{The acoustic power of radiation in a two-wall corner}

There are no real vibrating sound sources embedded only on the plane $x=0$ in the considered problem. Therefore, it is possible to obtain results valid for the two-wall corner directly from results presented herein for the three-wall corner by shifting the plane $x=0$ virtually to infinity (cf. Fig. 1). This can be achieved by the limiting transition $h_{12} \rightarrow \infty$, maintaining simultaneously the distribution of piston sources invariant with respect to one another, i.e. maintaining the distance $l_{12}$ invariant. Consequently, the distances $\left|x_{01}-x_{02}\right|, y_{01}$, and $z_{02}$ should also remain invariant. The limiting transition $d_{12}=h_{12} \rightarrow \infty$ is equivalent to $x_{01}+x_{02} \rightarrow \infty$, since $y_{01}, z_{02}=$ const. Therefore, virtual shifting of the baffle at $x=0$ to the infinity is equivalent to increasing the distance between the two real sources (the first piston and the second piston) and their two images to infinity.

In the case of small interference parameter values $k a_{1}$ and $k a_{2}$, i.e. when the linear sizes of the surface sources are small compared with the radiated wavelength $\lambda$, we obtain

$$
\begin{aligned}
& \lim _{h_{12} \rightarrow \infty} P^{\prime}(k)=2 k^{2} \frac{\sin k l_{12}}{k l_{12}}, \\
& \lim _{h_{12} \rightarrow \infty} P^{\prime \prime}(k)=2 k^{2} \frac{\cos k l_{12}}{k l_{12}},
\end{aligned}
$$

and (cf. Eq. (19))

$$
\lim _{h_{12} \rightarrow \infty} P=2 k^{2} \frac{\mathrm{e}^{\mathrm{i} k l_{12}}}{\mathrm{i} k l_{12}} .
$$

It is sufficient to neglect all the terms where cylindrical functions contain arguments depending on distances $x_{01}+x_{02}$ and $h_{12}$.

\section{The numerical analysis}

The total acoustic self-power of radiation of two vibrating circular pistons can be calculated numerically on the basis of Eqs. (1), (2a), (3a), and (A.1). For convenience, this quantity is formulated as the normalized total acoustic impedance of radiation

$$
\zeta=\theta-\mathrm{i} \chi
$$

where 


$$
\theta=\frac{\operatorname{Re} N}{N_{\text {Ref. }}} ; \quad \chi=\frac{-\operatorname{Im} N}{N_{\text {Ref. }}}
$$

are the normalized acoustic resistance and reactance of radiation, respectively. The following norm is used

$$
N_{\text {Ref. }}=\sum_{i=1}^{2} N_{i, \text { Ref. }} ; \quad N_{i, \text { Ref. }}=\frac{1}{2} \varrho_{0} c \int_{S_{i}}\left|v_{i}\right|^{2} \mathrm{~d} S_{i}
$$

which, in the case of a vibrating piston with a uniform distribution of the normal component of vibration velocity, simplifies to the form $N_{i \text {, Ref. }}=\frac{1}{2} \varrho_{0} c\left|v_{i}\right|^{2} S_{i}$.

Since both sources are located on two different walls of the three-wall corner (cf. Fig. 1), the effect of their acoustical mutual interactions on the total acoustic power of radiation is especially interesting. To analyze this effect it is assumed additionally that the square velocities over both sources are equal to one another, i.e. $N_{1 \text {, Ref. }}=$ $N_{2, \text { Ref. }}$. This leads to the expression $N_{\text {Ref. }}=\varrho_{0} c\left|v_{1}\right|^{2} S_{1}$ for the total reference power and allows focusing on the effect of such factors as the phase difference between the pistons' vibration amplitudes and their relative positions on the total acoustic power of radiation. The numerical analysis is performed assuming the following arbitrary values of the physical quantities: $c=340.0 \mathrm{~m} / \mathrm{s}$, $a_{1}=0.10 \mathrm{~m}, a_{2}=0.15 \mathrm{~m}, x_{01}=0.40 \mathrm{~m}, y_{01}=0.35 \mathrm{~m}$, and $z_{02}=0.30 \mathrm{~m}$. The values assumed for the remaining quantities are presented in Figs. 2 and 3. Three curves
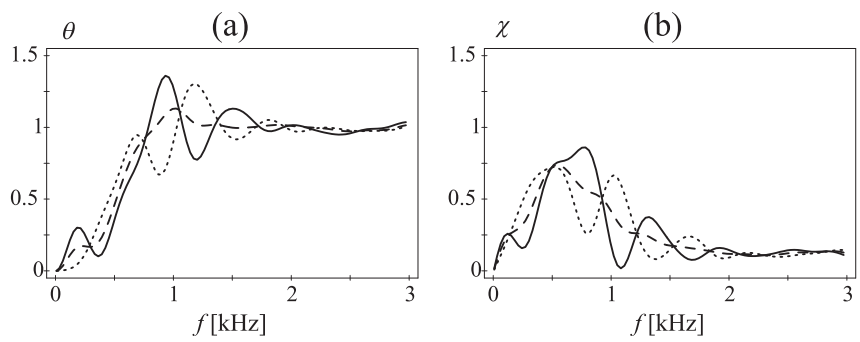

Fig. 2. Normalized active (a) and reactive (b) total acoustic power $\zeta=\theta-\mathrm{i} \chi$ for $x_{02}=0.25$ and different values of the phase difference $\delta$ : 0 (solid), $\pi / 2$ (dashed), and $\pi$ (dotted line).
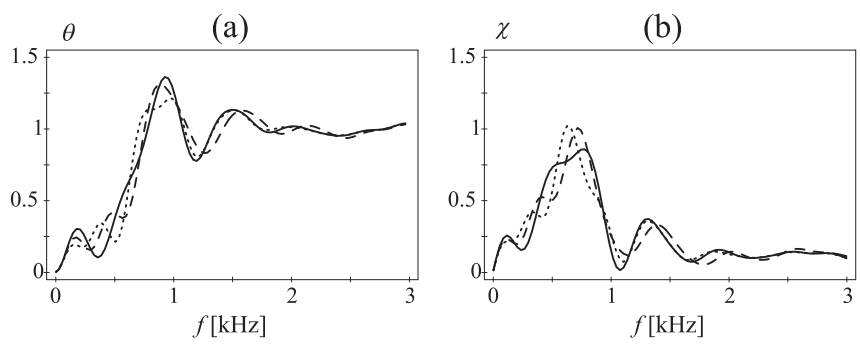

Fig. 3. Normalized active (a) and reactive (b) total acoustic power $\zeta=\theta-\mathrm{i} \chi$ for $\delta=0$ and different values of the distance $x_{02}: 0.25$ (solid), 0.40 (dashed), and 0.55 (dotted line).

presented in Fig. 2 are plotted for different values of the phase difference $\delta$ between the vibration amplitudes of the two pistons, namely $0, \pi / 2$, and $\pi$, for fixed locations of the pistons. The total acoustic power of radiation depends strongly on the phase difference $\delta$ within the frequency interval of about $0.0-2.0 \mathrm{kHz}$ which applies to the active acoustic power as well as to the reactive acoustic power. The acoustic power achieves its maximum value for the phase difference $\delta=0$ and the frequency $f \sim 1.0$ $\mathrm{kHz}$. The strongest attenuation of the acoustic power occurs for the same frequency and the phase difference $\delta=\pi$. The situation described above is reversed by increasing the frequency to about $1.2 \mathrm{kHz}$. With further increase in frequency, the active acoustic power tends to unity and the reactive acoustic power tends to zero which means that the acoustic mutual interactions of the pistons cease to affect the total acoustic power of radiation. The effect of the reflected acoustic waves also vanishes due to the elongation of distances related to the acoustic wavelengths.

The effect of the location change of one of the pistons on the total acoustic power of radiation, for a fixed value of the phase difference $\delta=0$, is presented in Fig. 3. The three curves are shown for different values of the distance $x_{02}$ between the center of the first source and the axis on which the second source's center is located. The effect is clearly visible within the frequency interval of about $0.0-2.0 \mathrm{kHz}$, although it is not as strong as this observed in Fig. 2.

The total active acoustic power of radiation achieves its maximum whereas the total reactive acoustic power achieves its minimum for the smallest value of $x_{02}$ and the frequency of about $1.0 \mathrm{kHz}$. Any change in frequency results in a significant change in the described situation. The effect vanishes completely with an increase in frequency over about $2.0 \mathrm{kHz}$.

\section{Concluding remarks}

The total acoustic power of radiation generated by two vibrating surface sources such as circular pistons located in the three-wall corner consists of such components as the acoustic self-power of the first source together with the acoustic power of radiation generated by three images of the first source representing the reflected acoustic waves, the acoustic self-power of the second source together with the acoustic power of the three corresponding images of the second source, the acoustic mutual power of radiation by the second source under the effect of the acoustic pressure exerted by the first source, and the acoustic mutual power of radiation by the first source under the effect of the acoustic pressure exerted by the second source (cf. the integral in Eq. (5a)). The normalized acoustic mutual power $n_{21}^{(-)}$presented in Eq. (5b) characterizes mutual interactions of the two sources, where $\left|x_{01}-x_{02}\right|$ and $r_{0}=\left[\left(x_{01}-x_{02}\right)^{2}+y_{01}^{2}\right]^{1 / 2}$ are the characteristic distances (cf. Fig. 1). The normalized mutual acoustic power $n_{21}^{(+)}$characterizes the effect of the acoustic pressure radiated by the first source on the second source in such a spatial configuration as the second source is located in the location of its image, so in 
the plane $y=0$. In the case of the normalized mutual acoustic power of radiation of the first source under the effect of the acoustic pressure exerted by the second source, the corresponding interactions are described by $n_{12}=n_{12}^{(-)}+n_{12}^{(+)}$where $r_{0}=\left[\left(x_{01}-x_{02}\right)^{2}+y_{01}^{2}\right]^{1 / 2}$ and $h_{0}=\left[\left(x_{01}+x_{02}\right)^{2}+y_{01}^{2}\right]^{1 / 2}$ are the characteristic distances.

The expansion series used for the reactive mutual acoustic power (Eqs. (10c), (10d) and (9)) are useful for numerical calculations. Eqs. (11g) and (13) are also worth noticing. They represent the expansion series containing the Bessel functions of the integer order and the associated Legendre polynomials.

In the case when the linear sizes of the piston sources are small compared with the radiated wavelength $\left(k a_{1}, k a_{2} \ll 1\right)$, their mutual interactions via the normalized mutual acoustic power are presented in their elementary forms. In the case of the active components, these are Eq. (7g) for $n_{21}^{\prime}$, Eq. (7h) for $n_{12}^{\prime}$, and Eq. (7i) for $n_{21}^{\prime}+n_{12}^{\prime}$. In the case of reactive components, this is Eq. (18) and Eq. (19) for the complex form.

The limiting transition is performed consisting in shifting the baffle with no real sources to infinity. As the result, a description of the acoustic power of radiation of the two pistons in the two-wall corner is obtained. Some of the reflected waves are removed and, as the consequence, the total acoustic power of radiation assumes smaller values in the two-wall corner compared with the values obtained in the three-wall corner. In the case of the two-wall corner, there is only one real source in the plane $z=0$. It has its central point at $O_{1}$ and one image with the center at $O_{1}^{\prime \prime}$. Significant differences of the acoustic mutual power of radiation in the two-wall corner and in the three-wall corner can be deduced from Eqs. (19) and (22).

The formulae presented herein are useful for low frequencies for the following two practical reasons. Firstly, the linear solutions presented provide a satisfactorily accurate approximation for small amplitudes of vibration velocities. Secondly, the most significant effect of the acoustic mutual interactions of the two vibrating pistons are observed for low frequencies.

\section{Appendix - The acoustic self-power of radiation}

The time-averaged acoustic self-power of radiation of the 1st source can be obtained from Eq. (1) assuming that $i=j=1$. It can be formulated as a single integral [19]

$$
\begin{gathered}
\frac{N_{1}}{\varrho_{0} c v_{1} v_{1}^{*} S_{1}}=f_{0}^{\infty} \frac{J_{1}^{2}\left(k a_{1} \tau\right)}{\tau \sqrt{1-\tau^{2}}}\left[1+J_{0}\left(2 k x_{01} \tau\right)\right. \\
\left.+J_{0}\left(2 k y_{01} \tau\right)+J_{0}\left(2 k \sqrt{x_{01}^{2}+y_{01}^{2}} \tau\right)\right] \mathrm{d} \tau
\end{gathered}
$$

after using the corresponding Green's function in its Fourier representation [18], where the quantities $2 x_{01}$, $2 y_{01}$, and $2\left(x_{01}^{2}+y_{01}^{2}\right)^{1 / 2}$ are the distances between the center of the 1st piston source and the centers of its three images which was discussed earlier in detail in [19].
The first component separated from Eq. (A.1) represents the self-power of radiation by the 1st source [26]

$$
\begin{aligned}
& \frac{N_{1}^{(1)}}{\varrho_{0} c v_{1} v_{1}^{*} S_{1}}=f_{0}^{\infty} \frac{J_{1}^{2}\left(k a_{1} \tau\right)}{\tau \sqrt{1-\tau^{2}}} \mathrm{~d} \tau \\
& =\frac{1}{2}\left[1-\frac{J_{1}\left(2 k a_{1}\right)}{k a_{1}}+\mathrm{i} \frac{\boldsymbol{H}_{1}\left(2 k a_{1}\right)}{k a_{1}}\right] .
\end{aligned}
$$

The remaining three components represents the acoustic power of radiation generated by the corresponding three images which can be expressed as [19]

$$
\frac{N_{1}^{(\mu)}}{\varrho_{0} c v_{1} v_{1}^{*} S_{1}}=f_{0}^{\infty} \frac{J_{1}^{2}\left(k a_{1} \tau\right)}{\tau \sqrt{1-\tau^{2}}} J_{0}\left(2 k l_{\mu, 1} \tau\right) \mathrm{d} \tau,
$$

where $\mu=2,3$, and $4 ; l_{2,1}=x_{01}, l_{3,1}=y_{01}$, and $l_{4,1}=\left(x_{01}^{2}+y_{01}^{2}\right)^{1 / 2}$. Further, the integral in Eq. (A.3) is expressed using the single expansion series [26]

$$
\begin{aligned}
& 2 \int_{0}^{\infty} \frac{J_{1}^{2}\left(k a_{1} \tau\right)}{\tau \sqrt{1-\tau^{2}}} J_{0}\left(2 k l_{\mu, 1} \tau\right) \mathrm{d} \tau=\sum_{s=0}^{\infty}\left(\frac{a_{1}}{2 l_{\mu, 1}}\right)^{s} \\
& \quad \times \sigma_{s}\left(k a_{1}\right) h_{s}^{(1)}\left(2 k l_{\mu, 1}\right)
\end{aligned}
$$

for $l_{\mu, 1} \geq a_{1}$, where $h_{s}^{(1)}(x)=j_{s}(x)+\mathrm{i} y_{s}(x)$ is the spherical Hankel function of the first kind and order $s$ [27], and the following coefficients are used [26]:

$$
\begin{aligned}
& \sigma_{0}\left(k a_{1}\right)=2 J_{1}^{2}\left(k a_{1}\right) \\
& \sigma_{1}\left(k a_{1}\right)=2 J_{1}\left(k a_{1}\right) J_{2}\left(k a_{1}\right) \\
& \vdots \\
& \sigma_{s}\left(k a_{1}\right)=\frac{2}{\sqrt{\pi}} \Gamma(s+1 / 2) \\
& \quad \times \sum_{m=0}^{s} \frac{J_{m+1}\left(k a_{1}\right) J_{s-m+1}\left(k a_{1}\right)}{m !(s-m) !} .
\end{aligned}
$$

Therefore, the total acoustic power of radiation of $i$ th source is expressed as the sum of four components $N_{1}=$ $N_{1}^{(1)}+\sum_{\mu=2}^{4} N_{1}^{(\mu)}$ in the case of acoustic radiation of only the 1st piston source in the three-wall corner. The acoustic self-power of the second source can obtained in a similar way.

\section{Acknowledgments}

The research presented in this paper was partially supported under The Centre for Innovation and Transfer of Natural Sciences and Engineering Knowledge Project at The University of Rzeszów.

\section{References}

[1] S.M. Hasheminejad, M.A. Alibakhshi, Arch. Acoust. 31, 243 (2006).

[2] F.G. Leppington, Q. J. Mech. Appl. Math. 20, 107 (1967).

[3] Y.S. Lee, H.J. Eom, Acta Acust. united Ac. 98, 365 (2012).

[4] H. Levine, F.G. Leppington, J. Sound. Vib. 121 , 269 (1988).

[5] N. Hashimoto, Appl. Acoust. 62, 429 (2001).

[6] J.P. Arenas, Int. J. Occup. Saf. Ergo. 15, 401 (2009). 
[7] J.P. Arenas, M.J. Crocker, Int. J. Acoust. Vib. 7, 217 (2002).

[8] Ł. Gorazd, J. Jurkiewicz, S. Raab, A. Snakowska, Acta Phys. Pol. A 123, 1085 (2013.

[9] A. Snakowska, J. Jurkiewicz, Acta Acust. united Ac. 96, $416(2010$.

[10] T. Mellow, J. Acoust. Soc. Am. 120, 90 (2006.

[11] J.P. Arenas, J. Comput. Acoust. 16(3), 321 (2008).

[12] L. Leniowska, Arch. Acoust. 33(4), 531 (2008).

[13] R. Trojanowski, J. Wiciak, Acta Phys. Pol. A 121, A148 (2012).

[14] M. Pawełczyk, Arch. Acoust. 33, 509 (2008).

[15] W.M. Zawieska, Int. J. Occup. Saf. Ergo. 13, 381 (2007).

[16] A. Brański, S. Szela, Arch. Acoust. 33, 521 (2008).

[17] S.M. Hasheminejad, M. Azarpeyvand, Shock Vib. 11, 625 (2004).

[18] W.J. Rdzanek, W.P. Rdzanek, Arch. Acoust. 31, 99 (2006).
[19] W.P. Rdzanek, K. Szemela, Arch. Acoust. 32, 339 (2007).

[20] W.P. Rdzanek, W.J. Rdzanek, K. Szemela, Arch. Acoust. 34, 75 (2009).

[21] W.P. Rdzanek, K. Szemela, D. Pieczonka, Arch. Acoust. 36, 121 (2011).

[22] K. Szemela, W.P. Rdzanek, D. Pieczonka, Acta Phys. Pol. A 119, 1050 (2011).

[23] I.S. Gradshteyn, I.M. Ryzhik, Table of Integrals, Series, and Products, Academic Press, 7 ed., 2007.

[24] B.G. Korenev, Bessel Functions and Their Applications, Analytical Methods and Special Functions, Taylor and Francis, London 2002.

[25] P.M. Morse, H. Feshbach, Methods of Theoretical Physics, Vol. 1 and 2, McGraw-Hill Book Company, New York 1954.

[26] R.L. Pritchard, J. Acoust. Soc. Am. 32, 730 (1960).

[27] G.N. Watson, A Treatise On The Theory Of Bessel Functions, Cambridge University Press, Cambridge 1944. 